\title{
SPINAL SPECIAL EDITION EDITORIAL
}

\author{
Andrew Quaile ${ }^{1}$
}

Published online: 19 May 2016

(C) SICOT aisbl 2016

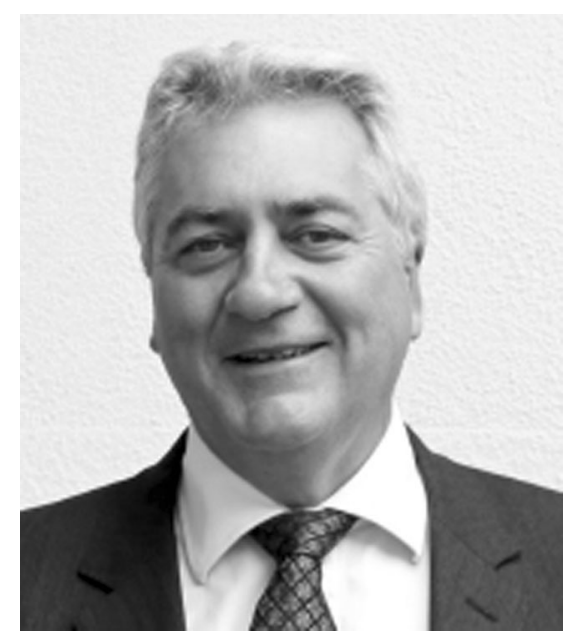

Andrew Quaile

I am honoured to be able to edit and comment upon this special edition related to spinal disorders. I am especially delighted that this edition covers a large range of operative and non-operative treatments in relation to spinal disorders and contains some really exciting developments in their treatment. I hope this special edition proves not only of great academic interest but also of practical value in treating your patients wherever you are throughout the world.

Over a career in medicine and orthopaedic surgery I have developed an interest in the treatment of spinal disorders. The treatment of spinal disorders is both challenging and complex. Over time techniques have come and gone or been refined.

Andrew Quaile

andrew@spine-works.com

1 Spineworks, Hampshire Clinic, Basingstoke, Hampshire, UK
The treatment of axial back pain via surgery is controversial, increasingly so in the conservative surgical environment of the United Kingdom. In contrast the treatment of neurological compression, caused by a number of pathologies, is less controversial and the treatment of deformity and trauma has become better understood and more effective with the advance of knowledge and technology.

During my career surgical techniques have evolved from the use of fixation by sublaminar wires by Luque rods and Hartshill rectangles. Sublaminar wiring, as a technique, still continues but has largely been superseded by the use of pedicle screws with various attachments. The use of pedicle screws was at first controversial and indeed at first during meetings in the United States cautions were given as their use had not been initially approved by the FDA. However they are now the main stay of attachment of instrumentation to the axial skeleton, especially in the treatment of fractures and deformity [1-9]. The last decade or two has also seen improvements in interbody cages and the re-emergence of anterior techniques to approach the spine [10,11]. Whether this is done by individual spinal surgeons, or assisted by an approach surgeon, depends upon the individual spinal surgeon's training and the institution in which they work. Posterior surgery has also advanced with the development of PLIF techniques but also TLIF techniques and XLIF techniques. All of these have been justified by their efficiency in placing the hardware and bone graft, or substitute, and with their apparent improved safety in such placement into the spine $[12,13]$.

More recently thoughts have turned to avoiding solid spinal fusion in axial back pain towards the soft stabilisations in order to limit pain caused by so-called spinal instability leading to reflex muscle spasm. These soft stabilisation were initially popularised by the GRAF technique with the GRAF ligament followed by the Dynesys technique. Both of these relied upon fixation to the skeleton via pedicle screws. After a 
surge of interest, the use of these techniques has declined to some extent although they are still used in some centres. Interlaminar techniques, for a combination of back pain and also in combination with decompression for neurological symptoms, remain popular. Indeed this is not new as spinous process plating was used in the treatment of unstable fractures many decades ago. The current interlaminar techniques are used to stabilise the spine and decompress the spinal canal either directly or indirectly. Direct implants, in association with spinal decompression, would involve devices such as the Coflex or Wallis ligament. Implants for indirect decompression of the spinal canal involve devices such as the XStop. A further novel approach for obtaining an axial fusion is the Axialif technique which continues to have some supporters, despite its possibly hazardous approach. More recently disc replacement has emerged as a viable technique, especially in the cervical spine [14].

One of the greatest changes during my career has been the ability to image the spine effectively. In a way this has raised a number of questions and still has not told us which disc or discs is painful in axial back pain. It is, however extremely useful in nerve root compression. It is also very useful in trauma [15]. There has been and equally encouraging advance in the so called 'percutaneous' techniques [16].

Prior to undertaking surgery, conservative options need to be considered. Indeed for the treatment of axial lower back pain spinal fusion surgery or disc replacement should be regarded as being the last option. During training conservative techniques were described to me as being either "supervised neglect or masterly inactivity" depending upon your point of view! Nowadays there are more scientific approaches via core stabilisation and postural retraining from expert physiotherapists. There is also a place for physical therapy from chiropractors and osteopaths. In regards to these allied professions, it has always been my view that it is not so much the discipline that counts but the individual clinician providing the therapy. Particularly in the treatment of back pain these allied practitioners can provide a valuable service in reassuring patients that the vast majority of their symptoms are not serious from a pathological point of view and are likely to settle in time and with the appropriate advice and support.

Non-operative pain control by injection has a major place in the armamentarium of the clinician treating spinal conditions [17]. In general the point of injections is to sufficiently reduce symptoms and break the downward spiral of pain and disability to allow further rehabilitation via physical therapy to take place. Clearly in most patients hydrocortisone injections would have a temporary effect but can be useful in allowing a window of opportunity for rehabilitation to take place. There was an enthusiasm for chymopapain to 'stabilise' the spine and reduce disc bulging however this was overtaken by modern methods.

Finally, and perhaps, most significantly, is the emergence of the potential that low back pain is associated with chronic infection and that antibiotics may be of benefit [18]. At the moment this is a concept and not yet proven. Nonetheless there are parallels with ulcer treatment where, when I was initially training, surgery had a major role. Indeed ulcer surgery was a major part of surgical training. The evidence at the moment for chronic infection is very limited and confined to a discrete group of patients developing modic changes after disc prolapse. Whether the use of antibiotics, with all the implications of long term antibiotic use, becomes useful in the long term is as yet unknown. There remains, of course the problem of infection in the spine whether or not associated with surgery. This can be a devastating complication [19-21] and other complications of surgery can be equally problematic [22-24].

The other potentially exciting area is the use of platelet rich plasma in axial back pain. There is indeed active research going on at the moment with the use of this technique in the treatment of axial low back pain. There is the potential that the use of this treatment would reduce the number of patients undergoing spinal fusions. This can only benefit patients in terms of reducing the collateral damage done to motion segments, not only at the operating site where a fusion has been obtained but also at the levels above and below. Significant cost reductions are also possible along with reduction of morbidity and complications. This, I believe, has an exciting future.

\section{Reference}

1. Jones et al (2016) Adolescent idiopathic scoliosis: evidence for intrinsic factors driving aetiology and progression. Int Orthop 40. doi:10.1007/S00264-016-3132-4

2. Hu et al (2016) Minimally invasive versus open transforaminal lumbar fusion: a systematic review of complications. Int Orthop 40. doi:10.1007/S00264-016-3153-Z

3. Lin et al (2016) Management of combined atlas-axis fractures: a review of forty one cases. Int Orthop 40. doi:10.1007/S00264-0153076-0

4. Popa et al (2016) Utility of the pedicle subtraction osteotomy for the correction of sagittal spine imbalance. Int Orthop 40. doi:10.1007/ S00264-016-3126-2

5. Zhang et al (2016) Limited long-segment fusion for degenerative lower lumbar scoliosis: a special kind of scoliosis. Int Orthop 40. doi:10.1007/S00264-016-3128-0

6. Zhang et al (2016) A comparison of three different surgical procedures in the treatment of type A thoracolumbar fractures: a randomized controlled trial. Int Orthop 40. doi:10.1007/ S00264-016-3129-Z

7. Ibrahim et al (2016) Mono segmental fixation of selected types of thoracic and lumbar fractures; a prospective study. Int Orthop 40. doi:10.1007/S00264-016-3152-0

8. Ma et al (2016) Effect of the percutaneous pedicle screw fixation at the fractured vertebra on the treatment of thoracolumbar fractures. Int Orthop 40. doi:10.1007/S00264-016-3156-9

9. Shchurova et al (2016) Operative management of high-grade dysplastic L5 spondylolisthesis with the use of external transpedicular fixation: advantages and drawbacks. Int Orthop 40. doi:10.1007/ S00264-016-3166-7 
10. Zhou et al (2016) Comparing the early efficacies of autologous bone grafting and interbody fusion cages for treating degenerative lumbar instability In patients of different ages. Int Orthop 40. doi: 10.1007/S00264-016-3124-4

11. Su et al (2016) Interspinous spacers versus posterior lumbar interbody fusion for degenerative lumbar spinal diseases: a metaanalysis of prospective studies. Int Orthop 40. doi:10.1007/S00264016-3139-x

12. Le Huec et al (2016) Bone morphogenetic protein use in spine surgery - complications and outcomes: a systematic review. Int Orthop 40. doi:10.1007/S00264-016-3149-8

13. Garin et al (2016) Natural hydroxyapatite as a bone graft extender for posterolateral spine arthrodesis. Int Orthop 40. doi:10.1007/ S00264-016-3140-4

14. Shen et al (2016) Clinical and radiological analysis of Bryan cervical disc arthroplasty: eight-year follow-up results compared with anterior cervical dlscectomy and fusion. Int Orthop 40. doi:10. 1007/S00264-015-3098-7

15. Rajasekaran et al (2016) Prediction of posterior ligamentous complex injury in thoracolumbar fractures using non-MRI imaging techniques. Int Orthop 40. doi:10.1007/S00264-016-3151-1

16. Cai et al (2016) Transforaminal percutaneous endoscopic discectomy in the treatment of far-lateral lumbar disc herniations in children. Int Orthop 40. doi:10.1007/S00264-016-3155-x
17. Sae-Jung et al (2016) Outcomes of lumbar facet syndrome treated with oral diclofenac or methylprednisolone facet injection: a randomized trial. Int Orthop 40. doi:10.1007/S00264-016-3154-y

18. Cao et al (2016) Overview: the role of Propionibacterium acnes in nonpyogenic intervertebral discs. Int Orthop 40. doi:10.1007/ S00264-016-3115-5

19. Rajasekaran et al (2016) Pyogenic lumbar spondylodiscitis treated with transforaminal lumbar interbody fusion: safety and outcomes.Int Orthop 40. doi:10.1007/S00264-015-3063-5

20. Kakutani et al (2016) Surgical debridement with retention of spinal instrumentation and long-term antimicrobial therapy for multidrugresistant surgical site infections after spinal surgery: a case series. Int Orthop 40. doi:10.1007/S00264-015-3073-3

21. Zhang et al (2016) One-stage posterior focus debridement, interbody graft using titanium mesh cages, posterior instrumentation and fusion in the surgical treatment of lumbo-sacral spinal tuberculosis in the aged. Int Orthop 40. doi:10.1007/S00264-016-3161-z

22. Chen et al (2016) Risk factors for bone cement leakage in percutaneous vertebroplasty: a retrospective study of four hundred and eighty five patients. Int Orthop 40. doi: 10.1007/S00264-015-3102-2

23. Kiely et al (2016) The incidence and risk factors for post-operative ileus after spinal fusion surgery: a multivariate analysis. Int Orthop 40. doi:10.1007/S00264-016-3148-9

24. Sarahrudi et al (2016) Tracheostomy following anterior cervical spine fusion in trauma patients. Int Orthop 40 